\title{
Intestinal pseudo-obstruction due to amyloidosis of the colon in association with an intestinal plasmacytoma
}

\author{
David Nicholl and Terry Jones ${ }^{1}$
}

Departments of Surgery and ${ }^{1}$ Pathology, Royal Shrewsbury Hospital North, Mytton Oak Road, Shrewsbury SY3 $8 B R, U K$

\begin{abstract}
Summary: A case of large bowel pseudo-obstruction due to colonic amyloidosis associated with an intestinal plasmacytoma is described. The association of an intestinal plasmacytoma with massive local amyloid deposition has not to our knowledge been previously reported.
\end{abstract}

\section{Introduction}

Amyloidosis is an extremely rare cause of megacolon and intestinal pseudo-obstruction. ${ }^{1,2}$ Plasmacytoma of the large intestine is also a rare entity with some 18 published cases. ${ }^{3}$ The association between caecal mucosal plasmacytoma and massive local deposition of amyloid with intestinal pseudo-obstruction has not, to our knowledge, been described previously.

\section{Case report}

A 70 year old retired gardener presented with a 13 day history of constipation and increasing abdominal distension. For several years he had suffered from frequent bowel action 2-5 times daily. His problem had previously been attributed to intestinal hurry, following a selective vagotomy and pyloroplasty 19 years earlier for recurrent duodenal ulceration.

On examination, he was dehydrated with a palpable mass in the left iliac fossa and clinical and radiobiological signs of intestinal obstruction. Full blood count, ESR, biochemical profile and chest $\mathrm{X}$-ray were normal. A provisional diagnosis of large bowel obstruction secondary to a mass in the descending colon was made.

At laparotomy, an acquired megacolon with a huge faecal mass, approximately the size of a melon, was found in the descending colon. The caecum looked necrotic. No tumour was found macroscopically and a subtotal colectomy with ileo-sigmoid anastomosis was performed.

Correspondence: T. Jones, M.R.C.Path. Accepted: 20 May 1991
The resected specimen consisted of $14 \mathrm{~cm}$ of terminal ileum and $78 \mathrm{~cm}$ of colon. The caecum was grossly dilated and the mucosal surface showed discrete areas of ulceration with the underlying muscle being extremely friable. The serosal surface showed foci of red/purple discoloration associated with the areas of mucosal ulceration. The remainder of the colon was also dilated but the only other areas of ulceration were small shallow mucosal ulcers in the sigmoid colon due to the impacted faecal mass at this site.

Sections from the caecum showed massive deposition of amyloid (Figure 1). There was complete replacement of the caecal wall by amyloid with only the mucosa being uninvolved. The sites with the greatest degree of amyloid corresponded to the areas thought to be necrotic macroscopically. The distribution was both perivascular and interstitial with, in the most severely involved areas, no muscle being identified between the sheets of amyloid. The amyloid stained strongly with Congo Red and methyl violet and showed typical apple-green birefringence. Smaller amounts of amyloid were identified in the thinned muscularis propria of the remainder of the colon but ganglion cells were present in normal numbers. The caecal mucosa was abnormal with a dense plasma cell infiltrate extending through the full thickness of the lamina propria into the superficial submucosa.

Although the intercrypt space was normal near the luminal surface, deeper in the mucosa the infiltrate became confluent and there was crypt separation. Cytologically the plasma cells showed only mild to moderate nuclear atypia (Figure 2). Immunophenotypic analysis of the plasma cells using monoclonal antibodies to immunoglobulin heavy and light chains was done using a standard 


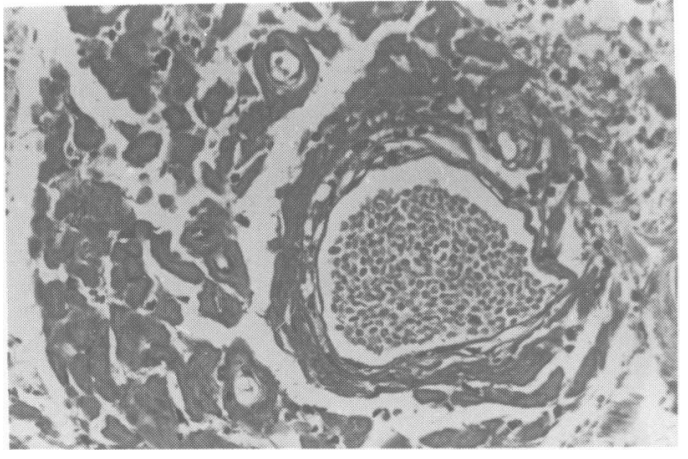

Figure 1 Colonic amyloid deposition. (Methyl Violet $\times 300$ ).

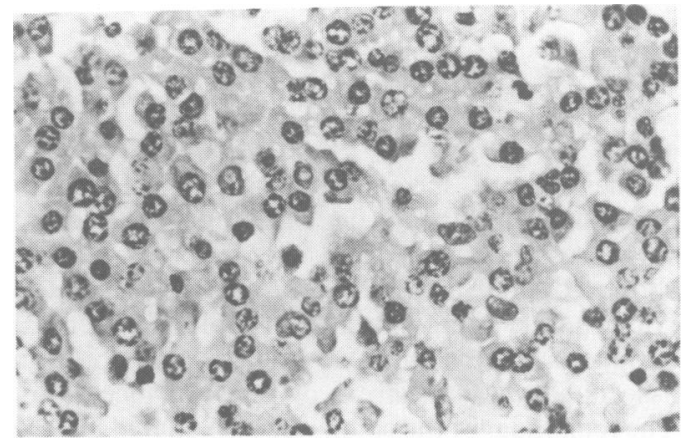

Figure 2 Plasma cells. (Haematoxylin and Eosin $\times 786)$.

avidin-biotin immunoperoxidase technique. This showed them to be expressing IgA with lambda light chain restriction indicating that they were monoclonal. Immunohistochemistry revealed the amyloid to be of an AL lambda type with negative staining for A protein.

Although no large discrete tumour mass was present, the diffuse monomorphous plasma cell infiltrate and light chain restriction indicated that this was an extramedullary caecal plasmacytoma. No lymphoepithelial lesions, such as small centrocyte-like cells infiltrating crypts, characteristic of other intestinal non-Hodgkin's lymphomas could be seen. The colonic lymph nodes identified showed reactive changes only. In view of these histological findings, a bone marrow aspiration and trephine biopsy, and skeletal survey were performed, which were normal, with no evidence of myeloma. Serum and urine electrophoresis were normal. Aspiration of the subcutaneous abdominal fat was negative for the presence of amyloid. He had an uneventful post-operative recovery and has remained well since.

\section{Discussion}

As many as $98 \%$ of patients with systemic amyloidosis also have involvement of the gastrointestinal tract. ${ }^{1}$ Hence, colonic amyloidosis usually occurs as part of generalized gastrointestinal involvement in the various known types of amyloidosis. Thus, in our patient it was important to look for evidence of systemic amyloidosis. Biopsy of the rectal submucosa has been traditional but also biopsy of other tissues, renal, liver, splenic and endomyocardial, has been used. Abdominal fat aspiration provides a high yield of positive results (up to $84 \%)^{4}$ with minimal risk. Scintigraphy with ${ }^{123}$ I-labelled serum amyloid $\mathbf{P}$ component where available may offer a non-invasive and highly sensitive technique for the evaluation of systemic amyloidosis. ${ }^{5}$

The majority of extramedullary plasmacytomas are found in the oral cavity and upper respiratory tract, rather than in the intestine. ${ }^{6}$ Plasmacytoma is most prevalent in the 40-70 year age group with a male-to-female ratio of $3: 1 .^{6}$ The diagnosis of an extramedullary plasmacytoma should only be made after excluding multiple myeloma by the histological findings, skeletal survey, bone marrow aspiration, and serum and urine electrophoresis. Several authors ${ }^{6-8}$ point out that some of the published cases have not in fact met all of these criteria.

In retrospect, this patient's prolonged history of diarrhoea suggests that the plasmacytoma with associated amyloidosis was a long-standing problem. There would appear to have been local secretion of a potently amyloidogenic light chain as a result of the plasmacytoma. Impaired motor activity with subsequent diarrhoea is believed to be a common presentation of colonic amyloidosis. It is thought that intestinal hyperactivity is a result of mechanical compression of nerve endings. Later, signs of obstruction can occur as a consequence of neuronal atrophy. ${ }^{1}$

In a similar case, an abdomino-perineal resection was performed on a patient with a solitary rectal tumour. ${ }^{9}$ Although there was no histological evidence of plasmacytoma, the immunochemical findings of AL-amyloid with lambda chains pointed to a plasmacytoma as the most likely underlying cause when the tumour recurred. Our case however demonstrates a more clear-cut histological association between a plasmacytoma and a large local deposition of amyloid in the large bowel.

\section{Acknowledgements}

The authors would like to thank Dr L. Englemann for translation of the German papers. 


\section{References}

1. Kumar, S.S., Samuel, S., Appavu, M.D., Abcarian, M.D. \& Barreta, T. Amyloidosis of the colon - report of a case and review of the literature. Dis Colon Rectum 1983, 26: 541-542.

2. Rewitzer, H., Wegmann, W. \& Stotz, R. Intestinal pseudoobstruction in primary amyloidosis of the intestinal tract. Schweiz Med Wochenschr 1984, 114: 1886-1890.

3. Price, A., Quilty, P.M. \& Ludgate, S.M. Extramedullary plasmacytoma of the rectum -2 cases treated by radiotherapy. Clin Radiol 1987, 38: 283-285.

4. Duston, M.A., Skinner, M., Shirahama, T. \& Cohen, A.S. Diagnosis of amyloidosis by abdominal fat aspiration. Am J Med 1987, 82: 412-414.

5. Hawkins, P.N., Lavender, J.P. \& Pepys, M.B. Evaluation of systemic amyloidosis by scintigraphy with I-labelled serum amyloid P component. $N$ Engl J Med 1990, 323: 508-513.
6. Asselah, F., Crow, J., Slavin, G., Sowter, G., Sheldon, C. \& Asselah, H. Solitary plasmacytoma of the intestine. Histopathol 1982, 6: 631-645.

7. Wing, E.J., Perchick, J. \& Hubbard, J. Solitary obstructing plasmacytoma of the colon. JAMA 1975, 233: 1298-1299.

8. Nielsen, S.M., Schenken, J.R. \& Cawley, L.P. Primary colonic plasmacytoma. Cancer 1972, 30: 261-267.

9. Dorner, O., Tolksdorff, G., Gamstatter, G., Bettendorf, U. \& Schmidt, H. Solitary primary amyloid tumour of the rectum. Leber Magen Darm 1989, 19: 95-96, 99. 